\title{
Home Security System Using Reconfigurable Robot
}

\author{
Gašparík Marek*, Šolek Peter \\ Institute of Applied Mechanics and Mechatronics, FME STU, Nám.slobody 17, 81231 Brat islava, Slovakia \\ *Corresponding author: mg.gasparik@gmail.com
}

Received October 07, 2014; Revised November 01, 2014; Accepted November 26, 2014

\begin{abstract}
Mobile robots are now widely used in various security and surveillance applications. The contribution describes a design of the robot with the function of the security system in the home. The robot is movable on wheels with various sensors, which sense the movement in the home. Based on detected movement will start tracking the object and shooting the camera. Picture and sound are transmitted to the mobile phone via 3G video call.
\end{abstract}

Keywords: robot, sensors, IR sensors, camera, security system, inthernet, GSM modem

Cite This Article: Gašparík Marek, and Šolek Peter, "Home Security System Using Reconfigurable Robot.” American Journal of Mechanical Engineering, vol. 2, no. 7 (2014): 303-306. doi: 10.12691/ajme-2-7-28.

\section{Introduction}

The target is to design and construct a robot whose function will be security system at home. Paper [1] presents of a novel wireless sensor network based home security system with a modular self-reconfigurable robot. Paper [2] describe the detection modules of the system which include active security modules and passive security modules. A circle object recognition method based on monocular vision [3] for the home security robots is proposed. The design and implementation of a new indoor [5] security system with a jumping robot as the surveillance terminal. The jumping robot a gateway and some pyroelectric infrared (PIR) sensor nodes form a ZigBee wireless sensor network (WSN). Paper [4] introduce a new human tracking system based on the fusion sensor consisting of the vision sensor and the depth sensor. In the paper [6] the mobile robot for collecting environmental information is applied to home automation. At the paper [7] when IA robot approaches an obstacle into defined distance ISR will start to avoid obstacle. Paper [8] propose home security system based on sensor network configured by sensor nodes including radio frequency, ultrasonic, temperature, light and sound sensors. Proposed home security robot [9] can detect abnormal and dangerous situations and notify user through internet and GSM modem. At the paper [10] develop a multiple remote interface security system (MRISS) that integrated with intelligent security robot, security supervise computer, GSM module, RF interface and appliance control module. The security robot [11] has a software development system, avoid obstacles and motion planning system, image system, sensor system remote supervise system and other systems. The intelligent security robot [12] can detect abnormal and dangerous situations and notify users. There is a software development system, avoiding obstacle and motion planning system, image system, sensor system, remote supervise system and other systems. The paradigm of robot [13] which performs specific tasks remotely has changed into intelligent robot to perform public and individual tasks.

The robot has to be controlled by mobile phone using DTMF or by RG system. In the case that the operator is unavailable the robot will work in autonomous mode. In this mode robot automatically react to stimuli from environment. The robot will broadcast audio and video via a mobile phone using $3 \mathrm{G}$ video call.

\section{Description of the Functions and Properties of the Robot}

Table 1 . Sensors in the security robot

\begin{tabular}{cc}
\multicolumn{2}{c}{ Table 1. Sensors in the security robot } \\
\hline Subsystem & Sensors \\
\hline Intruder detection subsystem & Ultrasonic sensors \\
& Body sensors \\
Environment detection subsystem & IR sensors \\
& Lux meter sensor \\
Obstacle detection subsystem & Voice sensor \\
& Distance Measuring Sensor \\
& IR sensors \\
Dynamic detection subsystem & Camera \\
& Gyro sensors \\
& Accelerometer sensors \\
Power detection subsystem & Compass sensors \\
& Current sensors \\
Motor control subsystem & Voltage sensors \\
\hline
\end{tabular}

The devices are shown in Table 1 and are shown in Figure 1. Robot is controlled by the built-in mobile phone for the purpose of transmitting control information over a long distance. Used is the phone C902 from SonyEricsson. If the $3 \mathrm{G}$ call is in activity with remote mobile phone, immediately is transmitted current image. Pressing the mobile phone keypad activate all control outputs to provide the movement of the robot. This feature allows you to track the object without external expressions. The robot behaves quietly in the relation to environment. The robot is controlled using the numeric keypad. Block diagram of the shooting environment of the robot with the function of the security system in the home is in Figure 1. 
Table 2. Type designation of sensors in the security robot

\begin{tabular}{cc}
\hline Sensors & Examples \\
\hline Body sensors & Switch \\
PIR sensors & IRA-E700ST0 \\
Lux meter sensor & FR12/100 \\
Voice sensor & Microphone \\
Distance Measuring Sensor & GP2Y0A41 \\
Ultrasonic sensors & HC-SR04 \\
IR sensors & TOSP4838 \\
Camera & Sony Ericsson C902 \\
Gyro sensors & L3G4200D \\
Accelerometer sensors & LSM303DLHC \\
Compass sensors & LSM303DLHC \\
Current sensors & ACS712ELCTR-05B \\
Voltage sensors & Voltagedivider \\
Incremental encoders & ED16112O \\
\hline The robot is controlled by the built-in RC
\end{tabular}

The robot is controlled by the built-in RC receiver. The receiver has six independent channels to control servomotors. The disadvantage of this controller is its maximum range. Audio video recording activated PIR sensor that provides information to the main processor, or in a secure area is occured motion. If so the camera starts recording. If in a secure area is not a man, after 10 seconds, the camera turns off and the record is saved in the SD memory card.

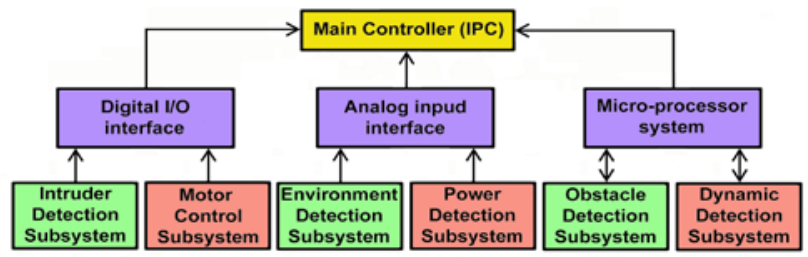

Figure 1. Engaging sensors in the robot

Mobile phone also uses a PIR sensor as with the audiovideo record. His activation and a function are the same, except that the combination of the key will send the last caller to the phone. Subsequently carried out the video call. This allows you to set from the distance on which phone number will robot call in case of disruption secured building. Pressing the item "Settings" in the navigation menu we have the bility to switched or don't switched manually functions gyroscope, sound, headlights robot, infrared light, infrared sensor and sensors of the robot chassis.

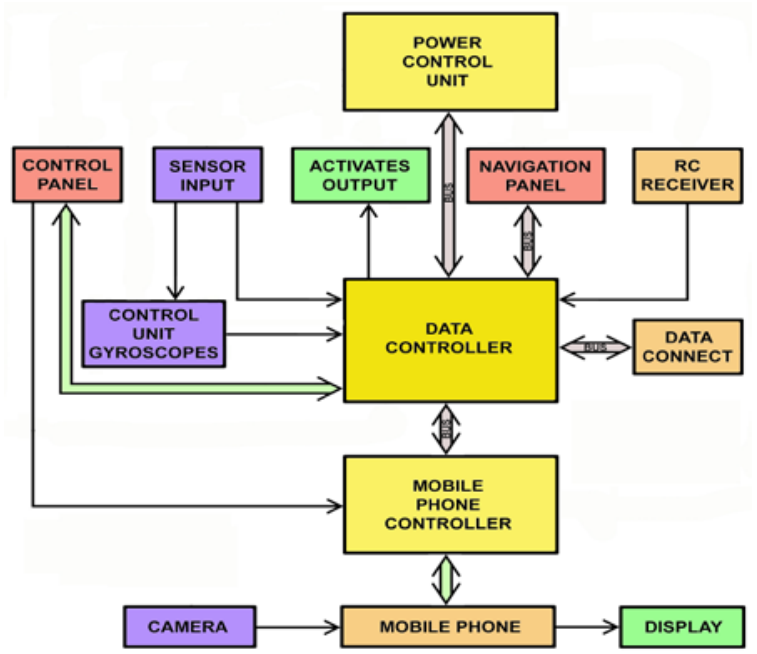

Figure 2. Block diagram of the robot with the function of a security system in the home

Block diagram of the robot with the function of a security system in the home is in Figure 2. Data controller communicates with the control units using I2C BUS. Power control unit supplied all adjacent systems. Its main task is to save energy. In the case of unnecessary control units, disconnect the power supply and significantly extend standby mode. Data Controller receives information from sensor inputs, control unit gyroscopes, control panel and then processing them according to the activated output. Mobile phone is controlled via optical members. These optical members are controlled with mobile phone controller that receives information using I2C BUS controller from Data controller.

\section{References Formats}

To get the smallest size of the robot, the robot with the whole frame and attachments has been drawn in CATIA. Furthermore, it was necessary to design a robot with the lowest weight and smallest size. This has been achieved in that the robot with the frame and attachments were modeled in full scale, to simulate the complete structure with the availability of each component of the difficult electronics. his approach has succeeded in reducing the weight of the robot frame from $320 \mathrm{~g}$ to $260 \mathrm{~g}$. At the same time was able to save the weight of one of the battery $60 \mathrm{~g}$. The result was a optimal robot, the practical implementation took place without a single change to the result of the design in CATIA.

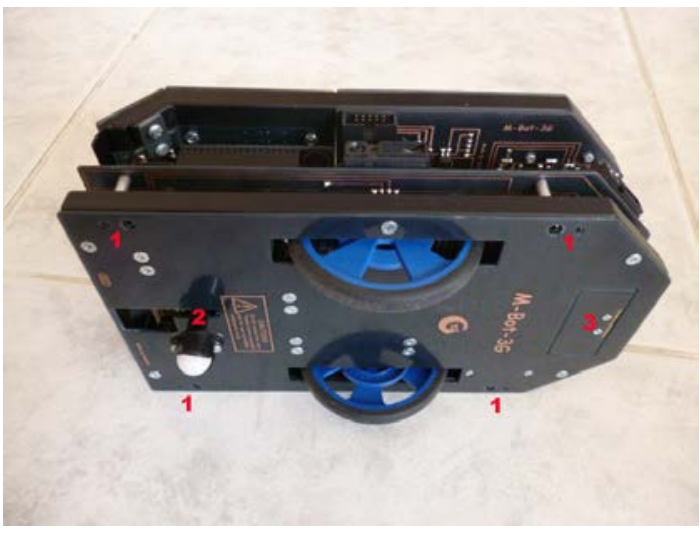

Figure 3. Main parts of the robot

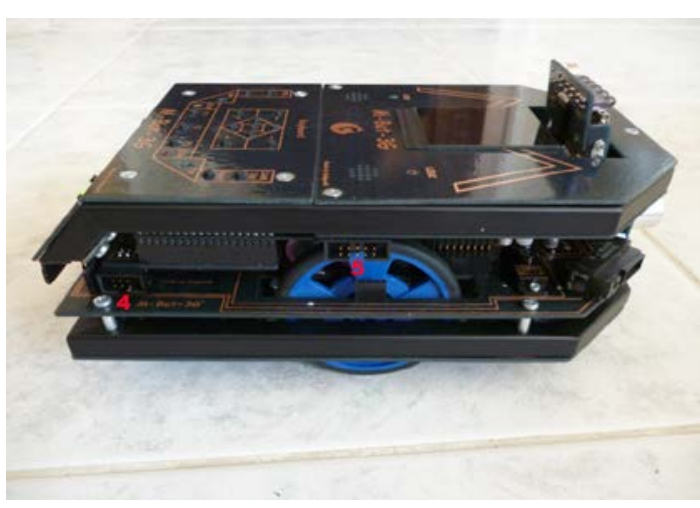

Figure 4. Main parts of the robot

Position 1 of Figure 3 describes the IR sensors, whose job is to scan the surface of the terrain surface and allow to assess whether its transition is possible or not. Position 2 of Figure 3 adjustable wheel support, which is designed to achieve stability of the robot. Position 3 of Figure 3 is the universal Hopper, which makes it a snap to different 
kinds of sensors. Position 4 Figure 4 is a connector that is used to program the robot. Position 5 of Figure 4 serves as a connector for an external keyboard.

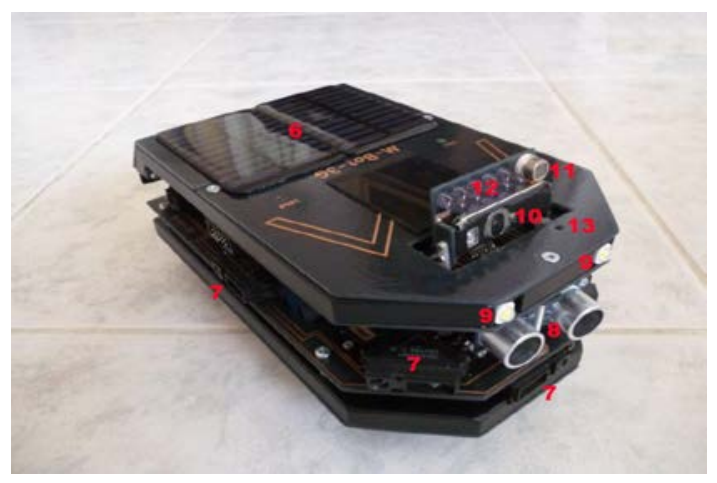

Figure 5. Main parts of the robot

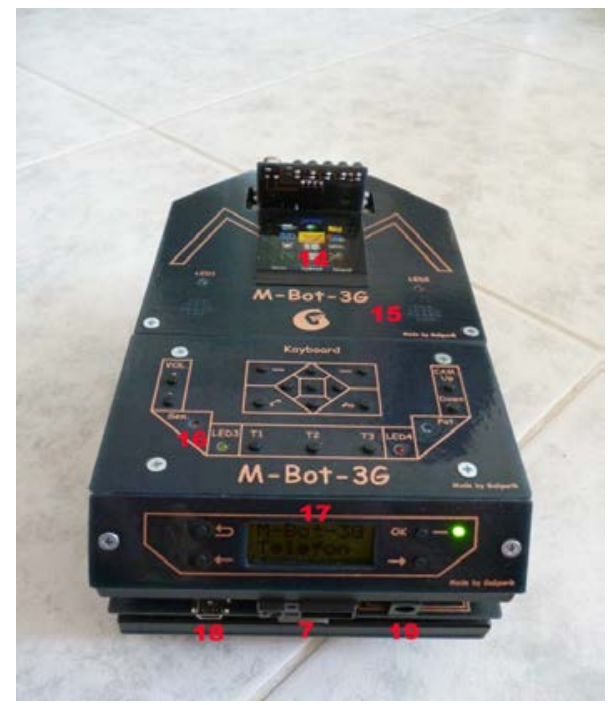

Figure 6. Main parts of the robot

Position 6 of Figure 5 are complementary solar panels. Position 7 of Figure 5 is IR distance sensors. Ultrasonic sensor position 8 is in Figure 5. Position 9 in Figure 5 are power LED diody. Position 10 in Figure 5 is a camera with automatic focus. Position 11 of Figure 5 is a PIR sensor. Infrared night vision is position 12 on Figure 5. It is the position 13 of the microphone in Figure 5. Position 14 of the Figure 6 is a graphical LCD display. Speakers at the Figure 6 are position 15. Position 16 of Figure 6 is a lux meter. Character LCD display is in Figure 6 position 17. Position 18 of Figure 6 is a USB interface and the position 19 on the Figure 6 is a charging connector.

\section{Design of the Security Robotic System}

Power is handled by two Lithium-Ion batteries connected in parallel. The block diagram of the robotic system is in Figure 2. Voltage is adjusted using four integrated circuits MC34063AP1 connected as a Step-Up. Integrated circuit MC34063AP1 voltage increases from 3.0 to $4.2 \mathrm{~V}$ to $5 \mathrm{~V}$, stabilized with $84 \%$ efficiency. Robot in Figure 5 has a number of active outputs that provide feedback in response to the environment. It hosts two cold-white LED with $150 \mathrm{~lm}$ luminous because of the good visibility in the dark. When observing an object in a dark environment, the camera is equipped with five pieces infradiods. The device contains five LED universal color information where their functionality depends on the software. For communicating audio messages there is the audio amplifier $2 \times 1 \mathrm{~W}$. Servomotors provide motion wheels, universal tipping platform, setting the third wheel support, control ultrasonic sensor and adjusting the height of the camera. The device was designed with versatile control via a microprocessor, which represents independent movement in space. Therefore, the proposed system external sensors that are in this kind of motion control necessary. These are optical, thermal (PIR), ultrasonic, position (gyro / accelerometer) sensors that give information about the obstacle position and then the signal is processed in the microprocessor as a command to change the direction of movement or perform the operation.
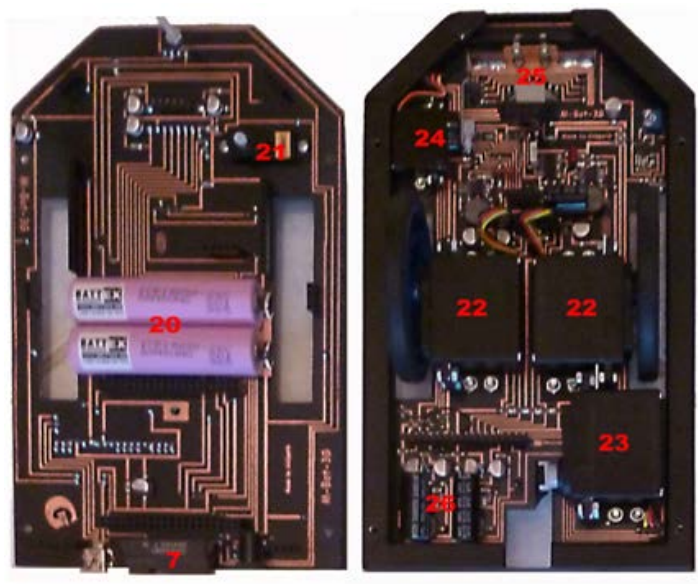

Figure 7. Main parts of the robot

Position 20 in the Figure 7 are the Li-ion batteries. Position 21 in Figure 7 is servo motor for movement of the ultrasonic sensor. The servo motor to drive the wheels is position 22 in Figure 7. Position 23 in Figure 7 is the servo motor to adjust the angle of the third support wheel. The Servo motor to move the hopper is position 24 in Figure 7. Position 25 in Figure 7 is universal hopper. Position 26 in Figure 7 are convertors.

\section{Conclusion}

The entire facility is designed to allow involvement exchange system control unit to manage the movement of universal robot. Thus, the method of control is changed, and the mechanical part is unchanged. This solution allows to continuously apply new kinds of management mode and so the entire device is actually useful in the next draft.

\section{Acknowledgement}

The authors would like to thank to the Ministry of Education of the Slovak Republic for supposting this research and for financial support here of under contract VEGA 1/0298/13.

\section{References}


[1] Qiao, G., Song, G., Wang, Y., et al, Autonomous network repairing of a home security system using modular selfreconfigurable robots, IEEE Trans. on Consumer Electronics, 59 (2013) 562-570.

[2] Chia, S.H., Guo, H., Li, B.Y., et al, Team mobile robots based intelligent security system, Applied mathematics \& information science, 7 (2013) 435-440.

[3] Li, S.A., Lin, Y.CH., Weng, CH.V., et al, Circle object recognition based on monocular vision for home security robot, Conf.IEEE ISPACS, New Taipei, 2012, 732-738.

[4] Jung, J.Y., Dan, B.K., An, K.H., et al, Real-time human tracking using fusion sensor for home security robot, Conf. IEEE ICCE, Las Vegas, 2012, 420-425.

[5] Zhang, J.S., Guangming, Q.G., et al, An indoor security system with jumping robot as the surveillance terminal, IEEE Trans. on consumer electronics, 57 (2011) 1774-1781.

[6] Lee, K.H., Seo, CH.J., Development of user friendly intelligent home robot focused on safety and security, Conf. IEEE ICCAS, Gyeonggi do, 2010, 389-392.
[7] Ren, C.L., Po, K.W., Yu, F.T., Navigation and mobile security system of home security robot, Proc. IEEE Int.Conf. on systems, man and cybernetics, 2006, 169-174.

[8] Kim, Y.G., Kim, H.K., Yoon, S.H., et al, Home security robot based on sensor network, Conf. SICE-ICASE, Busan, 2006, 18-21.

[9] Chia, S.H., Su, K.L., Chien, T.L., Develop an internet based home security robot, Conf. $12^{\text {th }}$ IASTED, Honolulu, Hidate, 2006, 14-16.

[10] Luo, R.C., Hsu, T.Y., Lin, T.Y., et al, The development of intelligent home security robot, Conf. IEEE ICN, 2005, 422-427.

[11] Luo, R.C., Su, K.L., Lin, K.C., Overview of the intelligent security robot Chung-Cheng, Conf. IEEE WARST,Nagoya, 2005, 220-225.

[12] Luo, R.C., Lin, T.Y., Su, K.L., Multisensor based security robot system for intelligent building, Robotics and autonomous systems, 57 (2009), 330-338.

[13] Ryu, J.G., Kil, S.K., Shim, H.M., et al, CDMA network operated mobile robot for security guard at home, Conf. IEEE ISI, San Diego, 2006, 633-638. 